\title{
QT Interval Prolongation Due to Right-Side Interscalene Block for Shoulder Surgery
}

\author{
Giuliano Bolondi', Francisco Javier Escribá Alepuz ${ }^{2}$, Russo Emanuele ${ }^{1}$, Domenico Pietro \\ Santonastaso ${ }^{1}$, Beatrice Benini ${ }^{1}$, Ana Maria Lopez Navarro ${ }^{3}$, Vanni Agnoletti ${ }^{1}$ and Pilar Argente \\ Navarro ${ }^{4}$ \\ ${ }^{1}$ Anesthesia and Intensive Care Unit, Ospedale Bufalini, Italy \\ ${ }^{2}$ Perioperative Medicine Group, Hospital Politecnico La Fe, Spain
}

${ }^{3}$ Unit of Anaesthesia and Reanimation, Hospital Intermutual de Levante, Spain

${ }^{4}$ Head of the Unit of Anaesthesia and Intensive Care, Hospital Politecnico La Fe, Spain

*Corresponding author: Francisco Javier Escribá Alepuz MD, Grupo de Medicina Perioperatoria, Instituto de Investigation

Sanitaria La Fe, Avinguda de Fernando Abril Martorell 106A, 46026 Valencia, Spain

\section{ARTICLE INFO}

Received: 慧 December 19, 2020

Published: 㥃 February 02, 2021

Citation: Giuliano Bolondi, Francisco Javier Escribá Alepuz, Russo Emanuele, Domenico Pietro Santonastaso, Beatrice Benini, et al. QT Interval Prolongation Due to Right-Side Interscalene Block for Shoulder Surgery. Biomed J Sci \& Tech Res 33(4)-2021. BJSTR. MS.ID.005425.

Keywords: Regional Anesthesia; Upper Extremity; Postoperative Pain; Interscalene Block; Shoulder Surgery; Electrocardiography; QT; Hemodynamics

Abbreviations: ECG: Electrocardiography; ERAS: Early Recovery After Surgery; GA: General Anesthesia; ISB: Interscalene Nerve Block; LCSD: Left Cardiac Sympathetic Denervation; LQTS: Long QT Syndrome; SG: Stellate Ganglion; US: Ultrasounds

\section{ABSTRACT}

Introduction: Inadvertent sympathectomy might lead to significant hemodynamic and electric instability. Sympathectomy can happen because of stellate ganglion block after interscalene nerve block (ISB) for shoulder surgery analgesia. In this study we aim to provide definitive evidence of QTc prolongation after right-ISB, thus leading to practical considerations that might affect daily clinical practice.

Material and Methods: A single center, non-blinded, perspective observational study has been performed. ECG analysis and QTc measurement were performed before and after 60 right-ISB and 47 left-ISB for shoulder surgery. Sub-analysis of the patients developing Horner syndrome was also performed.

Results: QTc results significantly prolonged after right-ISB (482 \pm 56 MS) with respect to the basal value of the same patients $\left(425 \pm 44 \mathrm{~ms}\right.$, $p$-value $\left.=3.68 \times 10^{-10}\right)$ and with respect to those patients receiving left-ISB $\left(420 \pm 41 \mathrm{~ms}\right.$, p-value $\left.=3.38 \times 10^{-8}\right)$. After right-ISB a higher proportion of patients (38 over 60) resulted with a dangerous QTc $\left(>450 \mathrm{~ms}\right.$ ) than after left-ISB ( 9 over $47, \mathrm{p}$-value $=2.07 \times 10^{-4}$ ). These observations were significantly more evident in the subgroup of patients developing Horner syndrome after ISB. Despite not reaching statistical significance, we observed that all clinically relevant intraoperative hypotension-bradycardia events happened in the right-ISB group and that after left-ISB a tendency in QTc shortening was present.

Conclusion: Right and left stellate ganglions have asymmetric sympathetic activity in regulating cardiac repolarization. Right-ISB patients are theoretically exposed to an increased risk of arrythmias and hemodynamic instability. This finding confirms important hypothesis on cardiac physiology and should lead to deeper preoperative evaluation and perioperative management also of low-risk candidates to shoulder surgery.

\section{Introduction}

Multimodal anesthesia and analgesia play a key role in the Early Recovery After Surgery (ERAS) protocols and locoregional anesthesia is a fundamental pilaster of this multimodal approach [1]. Locoregional techniques are widely applied, but their relatively recent birth is accompanied by the progressive description of previously unforeseen adverse effects that might in part modify their indications. Shoulder surgery is nowadays usually addressed through interscalene brachial plexus block (ISB). Despite ultrasound (US)-guided techniques allowing the injection of lower volumes of local anesthetic solutions, Horner syndrome due to stellate ganglion 
(SG) block, recurrent laryngeal nerve block and phrenic nerve palsy are frequently observed [2,3]: they are both expected side effects of this technique and predictors of block success. Bradycardia and hypotension are also frequently reported after ISB and general anaesthesia (GA) induction: venous pooling during beach-chair position [4] and Bezold-Jarisch reflex (an inhibitory vagal reflex possibly caused by peripheral nerve blockade in the neck) [5] have both been referred as possible causes, but also the side of ISB has been mildly associated with these hemodynamic symptoms [6]. SG is part of the sympathetic trunk. SG functional asymmetry in heart innervation has been proposed as a possible cause of long QTsyndrome (LQTS).

In fact, left cardiac sympathetic denervation (LCSD) has been surgically provided to treat unresponsive and life-threatening LQTS [7]. Moreover, electrocardiographic (ECG) modifications have been described after SG block [8,9]. Few years ago, Simeoforidou et al. [10] specifically studied a group of 24 patients undergoing shoulder surgery after ISB: probably due to the small sample size, they were only able to confirm slight ECG and hemodynamic differences between right- and left-sided ISB due to ipsilateral SG blockade. In our hypothesis, SG block can have a significant and frequently unrecognized effect on autonomic innervation, electrical heart activity and hemodynamic stability of patients undergoing elective shoulder surgery after ISB. This group of orthopedic patients comprehend a significant amount of young and sportive individuals that do not usually undergo instrumental preoperative evaluation (blood tests, ECG, ...), thus potentially exposing them to harmful cardiac events in case of unknown prolonged QT-interval. Our aim is to better define ECG modifications due to right- or left-ISB. These findings may definitively confirm the anatomic asymmetry of the autonomic nervous system regulating heart activity and can have a significant clinical impact, leading to different perioperative levels of alertness and loco-regional techniques depending on the side of surgery also for ASA 1 patients.

\section{Materials and Methods}

This observational prospective unblinded study has been conducted in a tertiary University Hospital along a period of 4 years, starting on February 2013, after the approval of the local ethical committee. Young healthy ASA 1 patients do not usually undergo ECG and analytic studies. Thus, following our usual preoperative path, preoperative anesthesiologic evaluation and ECG (allowing basal QT measurement) were performed to every man older than 45 and woman older than 50 candidates to arthroscopic surgery of the shoulder. During that visit, patients received information and a consent document on the study. Contraindications to perform of an ISB were coagulopathies, known allergy to local anesthetics, patient's refusal, infection of the puncture site, antecedent neuropathies or neurological problems. Exclusion criteria from the study were refusal to participate, intra- or postoperative uncontrolled pain (considered a sign of unsuccessful block), thyroid or renal disease-causing electrolytic disturbances, autonomic dysfunction, absence of preoperative ECG study, chronic arrhythmias and conduction disorders causing difficult or nonunivocal interpretation of the possible changes after ISB. On the day of surgery, participants accessed a quiet and warm preoperative room where basal monitoring (SpO2, non-invasive blood pressure and ECG) and a venous access were applied.

In-plane ultrasounds (US)-guided ISB was performed without neurostimulation by experienced anesthesiologists to conscious awake patients not receiving any sedation or premedication. Preceded by subcutaneous injection of $2 \mathrm{~mL}$ of Lidocaine $1 \%$ with a $25 \mathrm{G}$ needle, $20-25 \mathrm{~mL}$ of Ropivacaine $0,5 \%$ with a $22 \mathrm{G}$ needle 50 mm long were injected. 30 minutes after, we tested sensibility to puncture of C5-C6 dermatomes and motor test asking the patient to rise the anesthetized arm. Horner syndrome development, episodes of clinically relevant bradycardia (heart rate $<50 \mathrm{bpm}$ ) and hypotension (Systolic Blood Pressure decrease $>30 \mathrm{mmHg}$ than the baseline) were recorded. We also repeated a 12-leads ECG and measured QT interval. Patients were then transferred to the theatre where GA was induced, and the intervention was performed in beach-chair position. Both preoperative and post-ISB ECG were performed with a DRE True 12-channel ECG. QT interval was calculated following Bazzet's formula: $\mathrm{QTc}=\mathrm{QT}(\mathrm{MS}) / \sqrt{\mathrm{RR}}(\mathrm{s})$. Normal distribution of the data was confirmed with the ShapiroWilk test. Categorical data were compared through chi-squared analysis, while continuous numerical variables (QT differences between groups) were analyzed with t-Student analysis. Multiple testing correction following Holm's method was performed. Thus, two-sided p-value $<0.05$ was considered significant. Statistical analysis was performed using R 3.1.1 [11], data and scripts used for the statistical analysis are available in the supplementary material.

\section{Results}

Initially we divided the patients in 2 groups, left-sided ISB and right-sided ISB, and did not find any significant difference in terms of sex, age, height, weight, preoperative QTc value, chronic assumption of drugs known to enlarge QT interval and Horner syndrome incidence after ISB, thus confirming the basal comparability of these two groups (Table 1). We did not find a significant difference in the incidence of intraoperative bradycardia or hypotension, but it is worth to notice that these clinically relevant complications have been observed only in the right sided ISB group: probably, with larger samples, we would have observed a significant correlation with the ISB side. While pre-ISB QTc did not differ between the two groups, post-ISB QTc became significantly longer in right-ISB patients $(482 \pm 56 \mathrm{~ms})$ than in left-ISB patients $(420 \pm 41 \mathrm{~ms})$, with an adjusted $\mathrm{p}$-value $=3.38 \times 10-8$. In fact, only in the rightISB group the QTc significantly increased after the nerve block (pre-ISB QTc $=425 \pm 44 \mathrm{~ms}$, post-ISB QTc $=482 \pm 56 \mathrm{~ms}$, adjusted p-value $\left.=3.68 \times 10^{-10}\right)$, while left-ISB group did not modify its QTc with nerve block (adjusted p-value $=1$ ) (Figure 1). This resulted in 
a significantly higher number of patients developing high-risk QTc after the right-ISB (38 over 60 ) than after left-ISB ( 9 over 47 ), with an adjusted p-value of $2.07 \times 10^{-4}$, where high risk QTc was defined as equal or longer than $450 \mathrm{Ms}$

Table 1: Descriptive statistics of the two main groups (right-ISB, left-ISB) analyzed in our study.

\begin{tabular}{|c|c|c|c|}
\hline & Right-ISB & Left-ISB & adjusted $p$-value \\
\hline Number of Patients (N) & 60 & 47 & \\
\hline $\operatorname{sex}(M / F)$ & $34 / 20$ & $19 / 21$ & 1 \\
\hline age (years) & $50 \pm 13$ & $51 \pm 12$ & 1 \\
\hline height $(\mathrm{cm})$ & $173 \pm 9$ & $170 \pm 9$ & 1 \\
\hline weight (kg) & $82 \pm 10$ & $80 \pm 11$ & 1 \\
\hline pre-ISB QTc (MS) & $425 \pm 44$ & $431 \pm 42$ & 1 \\
\hline preoperative QTc-enlarging therapies & 2 & 1 & 1 \\
\hline post-ISB Horner syndrome (n, \%) & $34(57 \%)$ & $21(45 \%)$ & 1 \\
\hline Intraop. hypotension or bradycardia (n) & 3 & 0 & 1 \\
\hline post-ISB QTc (ms) & $482 \pm 56$ & $420 \pm 41$ & $3.38 \times 10-8$ \\
\hline post-ISB high risk QTc (n, \%) & $38(63 \%)$ & $9(19 \%)$ & $2.07 \times 10-4$ \\
\hline
\end{tabular}

Note: No statistically significant differences were highlighted before ISB, confirming the basal comparability of the two groups and the absence of confounding factors in our analysis. Hypotension is considered as systolic blood pressure $<70 \mathrm{mmHg}$, bradycardia as heart rate $<50 \mathrm{bpm}$, high risk QTc as QTc $\geq 450 \mathrm{~ms}$.

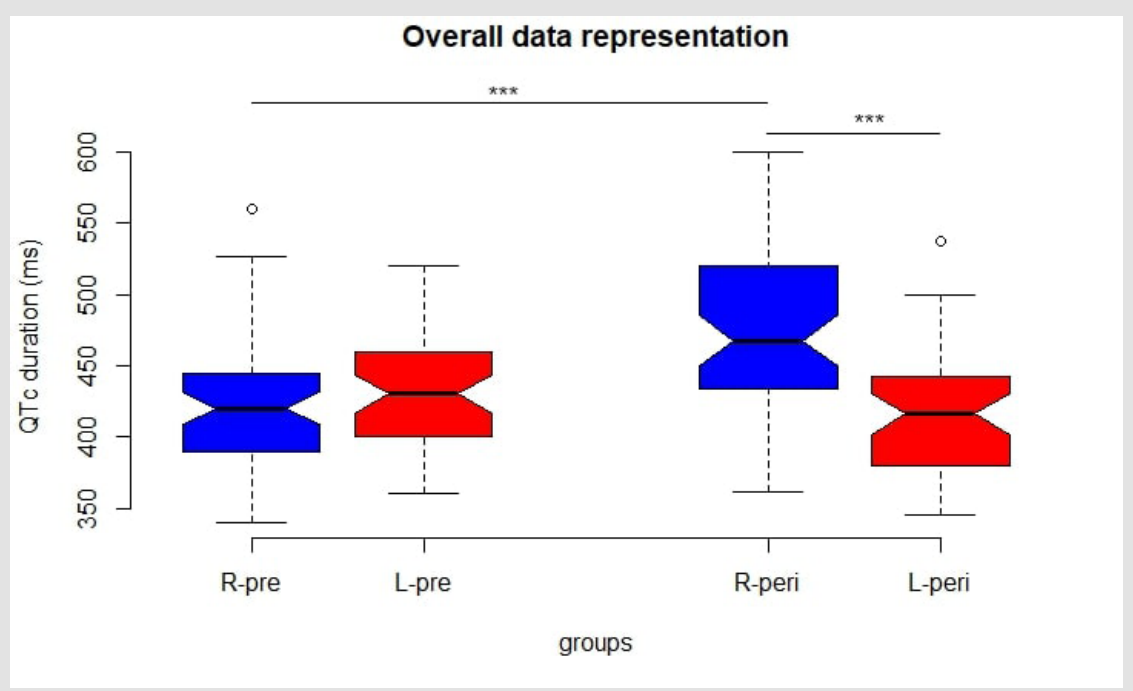

Figure 1: Representation of QTc length before and after ISB in left and right-sided ISB. R-pre: QTc duration before right-ISB, L-pre: QTc duration before left-ISB, R-peri: QTc duration 30 minutes after right-ISB, L-peri: QTc duration 30 minutes after leftISB, ${ }^{* * *}$ : statistically significant difference.

Successively, we analyzed the data of those patients developing Horner syndrome (Table 2). In fact, despite not observing any difference in the incidence of this phenomenon between left and right-ISB, it is an evident clinical phenomenon and, if demonstrated to be associated with dangerously enlarged QTc, it might be used as an alert for the anesthesiologists before the onset of sympatholytic or arrhythmic consequences. Patients developing Horner syndrome after nerve block had a similar QTc at the moment of preoperative evaluation (adjusted p-value $=1$ ) but developed a prolonged QTc after right-ISB (adjusted p-value $=4.19 \times 10^{-9}$ ) (Figure 2). In fact, patients developing Horner syndrome after right-ISB suffered a significant prolongation of QTc (pre-ISB $421 \pm 51 \mathrm{~ms}$, post-ISB $508 \pm 49 \mathrm{~ms}$, adjusted $\mathrm{p}$-value $=9.87 \times 10^{-12}$ ), while this did not happen in patients suffering Horner syndrome after left-ISB (preISB $443 \pm 49 \mathrm{~ms}$, post-ISB $407 \pm 43 \mathrm{~ms}$, adjusted p-value $=0.465$ ). Finally, patients developing Horner syndrome after right-ISB had a significantly longer QTc than those not developing the syndrome after same side block (right-ISB with Horner: $508 \pm 49 \mathrm{~ms}$, rightISB without Horner: $447 \pm 44 \mathrm{~ms}$, adjusted p-value $=8.34 \times 10^{-5}$ ). No significant QTc variations were observed after left-ISB when comparing patients developing or not Horner syndrome (adjusted p-value $=0.866$ ) . 
Table 2: Descriptive statistics of the patients developing Horner syndrome after ISB.

\begin{tabular}{|c|c|c|}
\hline & Right-ISB & Left-ISB \\
\hline Number of Patients (N) & 34 & 21 \\
\hline pre-ISB QTc (ms) & $421 \pm 51$ & $443 \pm 49$ \\
\hline post-ISB QTc (ms) & $508 \pm 49$ & $407 \pm 43$ \\
\hline ISB $=$ interscalene block. & & \\
\hline
\end{tabular}

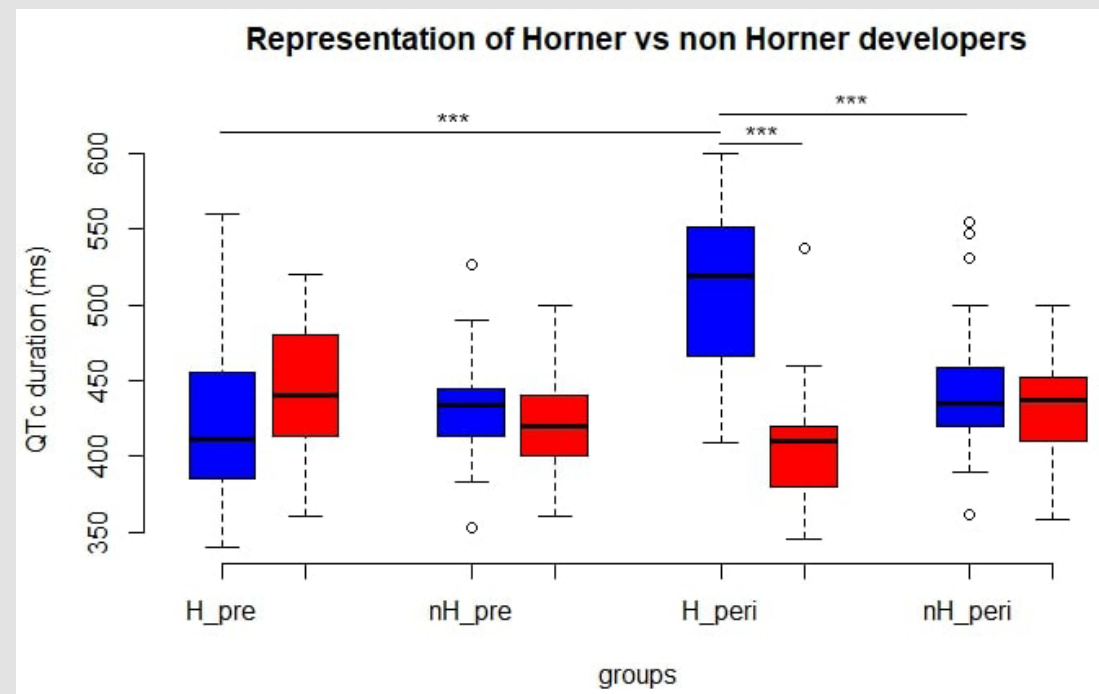

Figure 2: Sub-analysis comparing patients developing or not Horner syndrome, before and after ISB. Blue: right-ISB, red: leftISB, H_pre: QTc length before ISB in patients developing Horner syndrome, nH_pre: QTc length before ISB in patients not developing Horner, H_peri: QTc length 30 minutes after ISB in patients developing Horner, nH_peri: QTc length 30 minutes after ISB in patients not developing Horner.

\section{Discussion and Conclusion}

In this study, for the first time, we unequivocally demonstrate that right-ISB causes a significant prolongation of QTc up to dangerous levels. This phenomenon is even more evident in case of development of Horner syndrome: being clinically evident, this could be a clinical sign that should alert the anesthesiologist to strictly monitor patients developing it after right-ISB. These findings confirm the functional asymmetry of the autonomic nervous system regulating the heart and can be of clinical relevance for cardiologic and perioperative management of patients with long QTc. Despite not reaching clinical significance, we also observe a QTc reduction after left-ISB, confirming the observations leading to LCSD in the management of LQTS arrhythmic problems. We point out the need of a deeper preoperative study of patient's candidate to ISB because of the potential risk of long QTc-related arrhythmias and intraoperative hemodynamic instability. We think that, after these results, preoperative ECG study should be considered mandatory also in ASA1 and young patients undergoing shoulder surgery. The finding of a borderline or enlarged QTc interval should suggest avoiding right-ISB in favor of suprascapular [12] and/or circumflex $[13,14]$ block or intracapsular plus subacromial injection of local anesthetic [15] technique, that have been demonstrated to be equally effective in reducing intra and postoperative pain.
Moreover, in patients receiving right-ISB an extremely careful use of antiemetic drugs should be applied, knowing their tendency to prolong QT interval.

Thus, our results might tangibly vary the clinical management of these patients. The fact that the usual perioperative path has been used limits our study to males older than 45 and women older than 50 , potentially reducing the significance of our study in younger patients. Clinical significance in terms of hemodynamic and electric instability due to hypotension, bradycardia or longQTc related arrhythmias has also not been demonstrated, just remaining a theorical preoccupation, since non-significant results were obtained.

\section{Prior presentation}

The data reported in this paper have not been previously disclosed in other articles or poster presentation.

\section{Contributor Ship}

Dr. Escribà Alepuz conceived and designed the project, collected clinical data and informed consents.

Dr. Bolondi, Dr. Russo, Dr. Santonastaso and Dr. Benini analyzed, interpreted data and wrote the manuscript. 
Dr. Lopez Navarro collected clinical data and informed consents.

Dr. Argente Navarro and Dr. Agnoletti conceived and designed the project.

\section{References}

1. Ljungqvist O, Scott M, Fearon KC (2017) Enhanced Recovery After Surgery. JAMA Surg 152(3): 292-298.

2. Vester Andersen T, Christiansen C, Hansen A, Sorensen M, Meisler C (1981) Interscalene brachial plexus block:area of analgesia, complications, and blood concentrations of local anesthetics. Acta Anaesthesiol Scand 25(2): 81-84.

3. Borgeat A, Dullenkopf A, Ekatodramis G, Nagy L (2003) Evaluation of the lateral modified approach for continuous interscalene block after shoulder surgery. Anesthesiology 99(2): 436-442.

4. Shalev Y, Gal R, Tchou PJ, A J Anderson, B Avital, et al. (1991) Echocardiographic demonstration of decreased left ventricular dimensions and vigorous myocardial contraction during syncope induced by head-up tilt. J Am Coll Cardiol 18(3): 746-751.

5. D Alessio JG, Weller RS, Rosenblum M (1995) Activation of the BezoldJarisch reflex in the sitting position for shoulder arthroscopy using interscalene block. Anesth Analg 80(6): 1158-1162.

6. Seo KC, Park JS, Roh WS (2010) Factors contributing to episodes of bradycardia hypotension during shoulder arthroscopic surgery in the sitting position after interscalene block. Korean J Anesthesiol 58(1): 3844.

7. Schwartz PJ, Priori SG, Cerrone M, C Spazzolini, A Odero, et al. (2004) Left Cardiac Sympathetic Denervation in the Management of High-Risk

ISSN: 2574-1241

DOI: 10.26717/BJSTR.2021.33.005425

Francisco Javier Escribá Alepuz. Biomed J Sci \& Tech Res

(C) This work is licensed under Creative

Submission Link: https://biomedres.us/submit-manuscript.php
Patients Affected by the Long-QT Syndrome. Circulation 109(15): 18261833.

8. Egawa H, Okuda Y, Kitajima T, Minami J (2001) Assessment of QT interval and QT dispersion following stellate ganglion block using computerized measurements. Reg Anesth Pain Med 26(6): 539-544.

9. Saxena AK, Aggarwal B, Nakra D, Sethi AK, Aggarwal AN (2004) Evaluation of PR, RR, QT Intervals and QT Dispersion Following Stellate Ganglion Block in Chronic Shoulder-Hand Pain Patients. Pain Pract 4(2): 91-97.

10. Simeoforidou M, Vretzakis G, Chantzi E, M Bareka, K Tsiaka, et al. (2013) Effect of interscalene brachial plexus block on heart rate variability. Korean J Anesthesiol 64(5): 432-438.

11. (2014) R Core Team. R-Project.

12. Hussain N, Goldar G, Ragina N, Banfield L, Laffey JG, et al. (2017) Suprascapular and Interscalene Nerve Block for Shoulder Surgery: A Systematic Review and Meta-analysis. Anesthesiology 127(6): 9981013.

13. Rothe C, Asghar S, Andersen HL, Christensen JK, Lange KHW (2011) Ultrasound-guided block of the axillar nerve: a volunteer study of a new method. Acta Anaesthesiol Scand 55(5): 565-570.

14. Price DJ (2007) The Shoulder Block: A New Alternative to Interscalene Brachial Plexus Blockade for the Control of Postoperative Shoulder Pain. Anaesth Intensive Care 35(4): 575-581.

15. Fontana C, Di Donato A, Di Giacomo G, A Costantini, AD vita, et al. (2009) Postoperative analgesia for arthroscopic shoulder surgery: a prospective randomized controlled study of intraarticular, subacromial injection, interscalenic brachial plexus block and intraarticular plus subacromial injection efficacy. Eur J Anaesthesiol 26(8): 689-693.

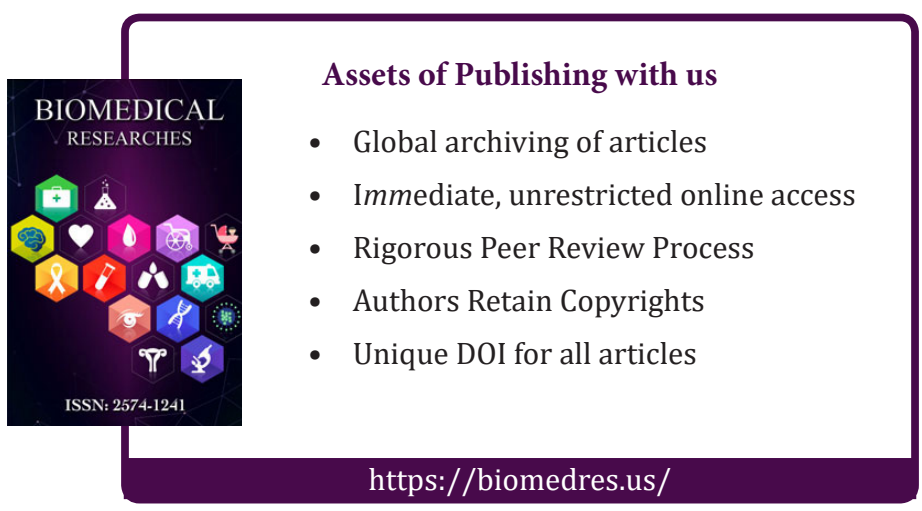

